This document is published in:

IEEE International Conference EDUCON: Proceedings of the 2012 IEEE Global Engineering Education Conference (EDUCON), Marrakech, Morocco 17-20 April 2012, IEEE, 6 p. DOI: 10.1109/EDUCON.2012.6201199

(c) 2012 IEEE. Personal use of this material is permitted. Permission from IEEE must be obtained for all other uses, in any current or future media, including reprinting/ republishing this material for advertising or promotional purposes, creating new collective works, for resale or redistribution to servers or lists, or reuse of any copyrighted component of this work in other works. 


\title{
m-Learning will Disrupt Educational Practices
}

\author{
Carlos Delgado Kloos, Raquel M. Crespo García, Carmen Fernández-Panadero, \\ $\mathrm{M}^{\mathrm{a}}$ Blanca Ibáñez, Mario Muñoz-Organero, Abelardo Pardo \\ Dep. Telematic Engineering, Univ. Carlos III de Madrid, 28911 Leganés (Madrid/Spain) \\ e-mail: $\{c d k$, rcrespo, mcfp, mbibanez, munozm, abel\}@it.uc3m.es
}

\begin{abstract}
In this paper, an overview is given about the research carried out in the area of mobile teaching and learning by Universidad Carlos III de Madrid, a member of the eMadrid Excellence Network. Mobile learning is raising growing expectations and is considered by some authors the next disruptive revolution in education. Cognitive and pedagogical theories supporting this prospect are reviewed. How these theories can be translated into meaningful educational practices is analysed by exploring ways of usage of mobile devices for supporting learning and teaching. Finally, a portfolio of experiments and case studies carried out by the Gradient group of the Universidad Carlos III de Madrid testing the application and effects of mobile learning are reported. These experiments show that the use of these devices is changing educational practices in a fundamental way.
\end{abstract}

Keywords-e-learning, technology-enhanced learning, mobile learning

\section{Mobile LEARNING: AN IMPORTANT Milestone}

New Information and Communication Technologies have always found very early application in the area of education [6]. When the computer was still an unconnected computing device, very soon multimedia presentations were produced that illustrated concepts in a visually appealing way. Also intelligent tutors helped to memorize and understand material. In parallel, communication technologies like radio and television allowed the creation of educational broadcasting channels. An important leap forward came when the computation and communication capabilities came together, especially with the internet and the web. This second milestone even enabled new business models for a new brand of distance universities and professional training centres. We believe that a third major milestone comes with having these computing and communicating devices in our pocket. Mobile devices will bring new educational possibilities to both students and teachers. They lower the access barrier to information to the minimum in any place at any time.

\section{COGNITIVE AND PEDAGOGICAL FOUNDATIONS}

According to Salomon [11] it is possible to extend human intelligence with intelligent technologies, People have been making machines more and more intelligent and now it is time to ask if machines can make people more intelligent, more specifically if increasing the use of mobile techniques can have a real impact in learning and cognitive processes.

\section{A. Extending the brain limits}

The information society overloads the knowledge workers, in particular students and teachers, with an immense amount of information. But the brain is limited both in short and in longterm memory. On the one hand, short-term memory is limited by the "magical number seven" [5] and can only hold around 7 pieces of information at a time. On the other hand, long-term memory is very effective at recognition of patterns in incoming information, but it is poor at recalling it (reviving memory patterns without perceptual stimulation).

This situation makes it necessary to off-load part of information and processing capabilities from our brain to external "cognitive artefacts" in the form of physical (sticky notes, file folders) or digital supports (computers, handheld devices). Mobile devices can combine of both supports, because they can deal with the digital world and also with the physical world by making use of augmented reality techniques.

But mobile devices do not only extend our brain in terms of memory and processing capability, they also extend our perceptions with sensors (camera, position, acceleration, tagscanners for augmented reality) and our ability to actuate in our environment (actuators). Being always connected, we can interact with the environment and other agents (people or devices) at any time in any place.

Thus, persons equipped with a mobile device enhance themselves with better cognitive abilities to perceive processes and appropriately respond to the challenges and affordances of their environmental situation.

\section{B. Pedagogical and cognitive procese-}

According to Heylighen [3], initially the guiding metaphor for analyzing cognitive processes was "symbolic cognition". In this paradigm, knowledge was an internal representation of the external environment based on symbols. But relevant aspects of the real world are too complex to be represented with symbols. This approach has been questioned very much in favour of other cognitive paradigms that are more inspired on the biological and neurological functioning of the mind, such as "situated and embodied cognition". This new approach argues that our actions are much more influenced by external conditions (situated) and our perception of it (embodied) than by reasoning and planning using our internal representations of knowledge.

In this approach the cognitive system, such as the human mind interacting with its environmental situation performing 
actions and monitoring their results via perceptions. This interaction is controlled via sensory-motor feedback.

Introducing hand-held devices in the learning process change the representation of the system. Now the cognitive system is not the student by itself but rather the complex system formed by the student and their external brains (mobile device + augmented environment)

- $\quad$ Perception trigger actions and our handheld device provide augmented perception of the reality so we have more information to trigger actions.

- Actions produce changes in the environment but these changes are not only in our immediate environment. Actions of mobile-enhanced people have much more impact because due to the connection capabilities of their devices can influence other agents (devices or people) in any place and at any time

- Changes are again perceived with our augmented perception due to our mobile device

- New perceptions trigger new actions, and mobile phones provide new triggers based on context, location, near devices, etc.

\section{Short-term and long-term effects of m-learning}

Salomon [11] distinguishes between short-term effects "with" the technology and long-term effects "of" the technology.

Short-term effects "with" technology can redefine and enhance performance as students work in partnership with technology that undertakes part of the cognitive process. For example, the burden of our short-term memory can be reduced using mobile devices as external memory to off-load important part of incoming information in digital form. The recall function of long-term memory can be improved triggering some reminders according to the date, the physical location (geo-location) or the context (people or devices in our near environment). The pattern recognition function of our longterm memory can be improved annotating resources and using searching, filtering, and processing capabilities of our mobile devices. We can also extend our perceptions and ability to interact with the environment with mobile sensors and actuators.

On the other hand, long-term effects "of" the technology occur when partnership with a technology leaves a cognitive residue, equipping people with new ways of doing things, even away from the technology.

The ability of mobile devices to monitor and register interaction and the ability of the user to share information facilitates the process to provide feedback for reflective thinking [6]. The interaction can be registered and monitored leaving some marks in the environment as a stigmergic process. And this information can be analyzed to obtain emergent properties and behaviours that can be reinforced or extinguished in terms of their usefulness for the user.
Although more simple long-term benefits such as our ability to gather, classify, or share information at any time in any place, can occur spontaneously as technology advances, more complex long-term benefits, such as new thinking skills and strategies to reorganize and enhance student performance are not likely to occur. These skills must be cultivated through new learning design, promoting new uses of mobile technologies in our learning environments. Section III provides some ways of usage that could be seen as short-term benefits, and section IV provides some study cases and more complex experiments using mobile devices for educational purposes that could be repeated to reach long-term benefits.

\section{WAYS OF USAGE}

There are many ways in which mobile devices can be used. In the following, we highlight a few of them.

\section{A. Augmenting student learning capacity}

The learning process involves the efficient use of human skills such as memorization, reasoning, inference, deduction, knowledge creation and sharing or inter-personal skills. Mobile devices can be used to extend and augment these learner skills in different ways:

- Calendar-like applications combined with location sensors such as GPS and activity detection through mobile device sensors and personal agents may be used to optimize the time utilization for the student.

- Voice and video recording capabilities in mobile devices can capture information to augment student data retention or to improve certain capabilities. For instance, Gromik [1] reports about an experiment where students would record themselves reading in a foreign language in order improve oral expression.

- Proactive personal agents running on a mobile device can be used to present information of interest to the learner either because of the detection of broadcasted information beacons (available remotely or locally using protocols such as HTTP, RSS, local BT broadcasting or social networking) or because it can be inferred by a personal agent.

- Wideband always available connectivity provides unlimited access not only to information resources but to relevant people for the learning process: other students, professors and experts. Mobile devices therefore augment communication channels for learners minimizing both distance and time physical constrains.

- Augmented reality interfaces through mobile devices can overlap information of interest to what the learner sees in the physical world. 


\section{B. Enhancing the learner experience}

Mobile learning offers the possibility to transform students from content consumers to content producers. They might explore or produce knowledge in a collaborative way. Besides the web2.0-based techniques to produce knowledge collaboratively, students now have the possibility to do it in a ubiquitous way. Any real object might have digital content to be explored and can be enhanced with multimodal information that students can attach to it.

Creativity will be fostered by the use of augmented reality (AR) technologies as support of creativity processes. AR might support divergent creativity techniques such as brainstorming techniques to generate news ideas and also convergent creativity techniques, such as highlighting and mind mapping. Divergent ideas can be generated by superposing digital information to real objects; the process can be done in a collaborative way fostering the imagination of peers. The complementary synthesis step can be done by combining or clustering the ideas previously generated. By supporting divergent and convergent mechanisms of creative processing, students will increase their capacity to create new knowledge and to solve more challenging problems.

\section{New ways of assessment}

Mobile devices are especially suited to easily extend assessment beyond the current scenarios. Students carry these devices in situations where learning can take place, but there is not an easy method to monitor the activity. For example, activities in various spatial locations can be used to design innovative learning scenarios. Mobile devices are privileged in this environment, because students are already used to their presence and they can be used to collect detailed information. When combining these newly emergent scenarios with the two types of assessment (formative and summative), mobile devices have great potential to improve how activities are assessed. Mobile devices already collect detailed information about the type of applications being used, the location, the persons interacting, etc. All these factors may contribute to a more accurate assessment of factors such as the level of interaction among team members, the amount of material used, the locations visited, the space/time relation with respect to a scenario, etc.

\section{Supporting other stakeholders}

The previous subsections discuss the use of mobile learning focused on the learner. However, we understand m-learning in the widest possible way. And, in a similar way as discussed for the students, mobile devices can support and complement the teachers' activity or even other stakeholders.

Teachers can also benefit from the abilities extension provided by mobile devices. Like students, teachers can also enjoy the advantages related to for example the facility of creating multimedia content (photography, video, audio, etc.) and permanent and ubiquitous connection. However, apart from the applications previously commented for students, which can naturally apply to the teachers' activity, some specifics should be mentioned. Storage facilities provided by mobile devices can support scaffolding and assessment, providing a complete and up-to-date report of the student's evaluation status, easy to update anywhere, anytime with new observations. Combined with augmented reality, this can help the teacher to identify students in mass classes and provide them with personalized feedback and support. In this line, the class-on system has been developed for providing awareness to the teacher and supporting students' assessment and the provision of quick feedback and notifications [2].

And beyond students and teachers, mobile devices can help to widen the learning process and open it to involve additional stakeholders who can contribute to improve its effectiveness. For example, in primary education, parents participate closely in the education of their children. Some projects have thus been developed to boost their motivation and encourage their involvement.

\section{EXPERIMENTS}

In this section, we describe several experiments carried out in our department, either in our own teaching classes or in outside ones. They intend to be a sampler of different uses of mobile devices for educational purposes.

\section{A. Creating a backchannel}

The mobile device allows creating an additional communication channel between the teacher and the student in class. In the same way, as we start seeing in some conferences that the audience tweets some impressions about the speaker and in this way all get enriched about new perspectives without disturbing the main talk, mobile devices can be used in class to improve the learning experience. One experiment we have done, consisted in giving the students mobile devices, where the students could report about two items:

- The teacher is too fast/too slow

- I am lost/I am following

The teacher could see on another similar mobile device the mean value of the student data. These pieces of information could be transmitted in a non-intrusive way.

For this experiment we used Maemo devices of Nokia (see figure 1). The students were given the devices during class. The teacher used the same device for monitoring the average values. 


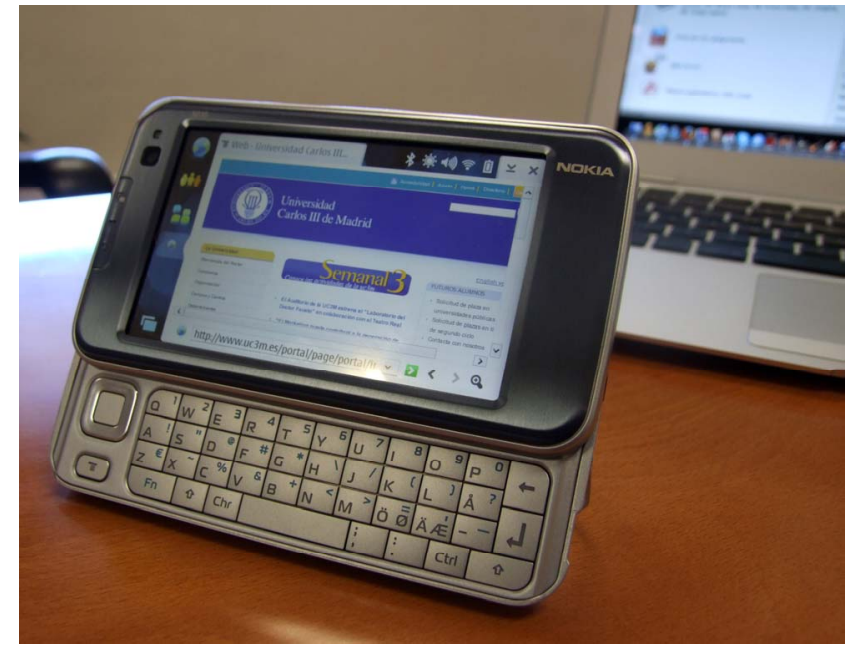

Figure 1. Mobile device used to create a backchannel

The same devices could were also used to let the students respond simple questions. This is the purpose of Audience Response Systems (ARS), which typically also provide some nice statistics about the responses. But there is no need any more to use these devoted tools. General-purpose mobile devices are much more flexible than the expensive specialized ARS tools that can be found on the market.

\section{B. Sending learning pills to students}

An experiment was carried out that was able to improve student class attendance, performance, and motivational patterns by using mobile devices in class to provide students with contextualized learning pills [7]. A learning pill is a simple exercise that summarizes some of the key concepts explained in class promoting reflection and self-study. Each student attending a particular lecture or laboratory session automatically received a learning pill on his or her mobile phone just after the related concept has been presented.

The rate of students attending more than half of the classes was slightly improved, by $3.5 \%$, comparing the offering of the course in which the learning pills were used to that in which they were not. The average grades for students attending classes in offerings of the course in which the pills were not used are significantly worse (using statistical significance) than those of students attending classes in which pills were sent. Finally, the use of learning pills has a positive impact on the evolution of the motivational states of the students over the duration of the course. This was especially true for intrinsic motivation, while being less noticeable for external motivation.

In this experiment, the students used their own devices. Therefore, no particular platform could be assumed. Bluetooth was used to send the learning pills, which is a very basic feature that can be assumed for most mobile phones. Figure 2 shows an example of learning pill sent to students.
- Replace the XXX in the figure and describe the process:
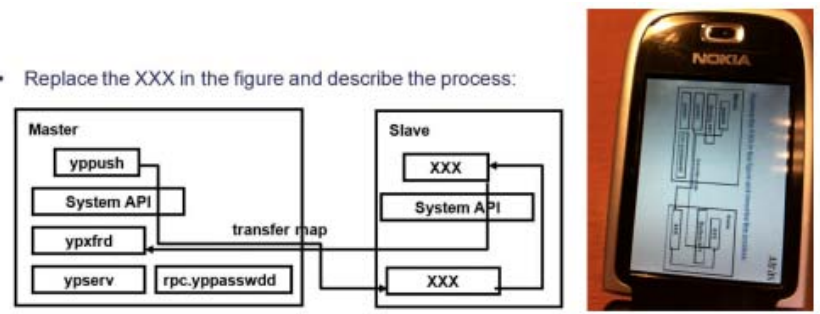

Figure 2. Example of learning pill sent to students

\section{Educational gymkhanas}

When freshmen come to university have often have difficulties in learning where things are. It is useful to help them in exploring the campus. Together with colleagues from the Universitat Pompeu Fabra (UPF), we have developed a setting that allowed new students to explore the UPC campus [8] (see figure 3). NFC Mobile phones and NFC tags were the support for the campus exploration. Software tools were developed for writing and reading the tags. Bluetooth technologies were used to collect the log files resulting from the exploratory activity. Finally, Moodle and Google Docs were the web-based software tools employed for editing and answering the questionnaires and for uploading and sharing the final works.

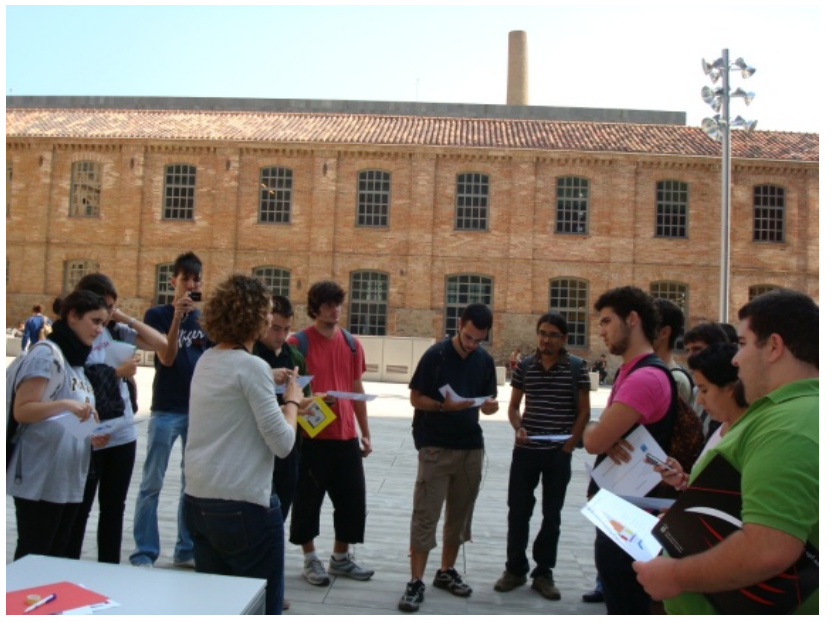

Figure 3. Exploring the campus at Universitat Pompeu Fabra

In this way, mobile devices were a permanent companion to the students in their discovery of the campus, a discovery that could be monitored and screened, in order to improve the experience on later occasions.

\section{Augmented reality}

A marker-based game for children was developed that consisted of combining syllables to compose as much words as possible [9]. Syllables were coded in fiduciary markers so that the player can handle them, as if they were counters of some traditional game. The Augmented Reality engine (AMIRE in this case) watches the playing scenario and detects the correct combination when the player puts the right syllables together. Automatically, the application sticks a 3D model that represents the word on top of the markers (see figure 4). 


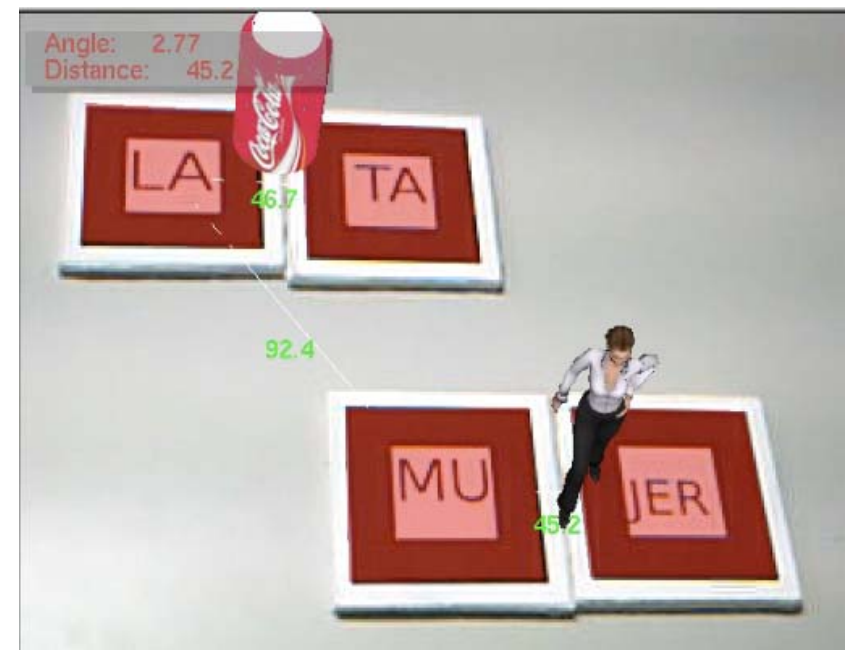

Figure 4. Example of the use of Augmented Reality to learn syllables

The role of AR in this game is to provide feedback to the user and enhance the association of concepts. This game does not allow teaching abstract names.

Several other games were developed in order to learn the basic arithmetic operations or to be used as augmented flashcards (see [9] for more information).

\section{E. Cross reality}

Immersive 3D virtual world platforms are powerful tools where persons can be immersed through their avatars in imaginary or mirror worlds and interact in a variety of ways. Their use in education is multiple [4], but it normally requires sitting in front of a computer. Nevertheless several other uses are possible without using a big-sized monitor. One possibility is to use special glasses, but this ties the person too much to the computer.

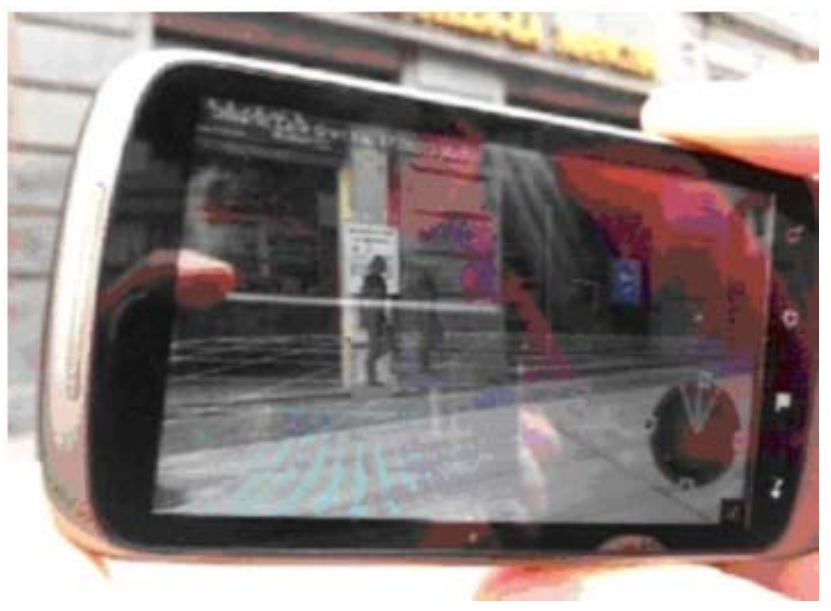

Figure 5. Cross reality with avatars reflected from a virtual world superimposed on an image coming from the real world

What we have been developing is a combination of $3 \mathrm{D}$ worlds and augmented reality with a mobile device. We enrich the physical reality that comes through the screen of the mobile device with information coming from a mirror $3 \mathrm{D}$ world, i.e. a
3D virtual world that mirrors the real one with authentically geo-localized buildings. In our mobile phone we can see the positions of the avatars superimposed on the image of the physical world (see figure 5). We can also send text, voice, or images from the physical to the virtual world and vice versa.

We can imagine many applications for this mixed reality application, which would be impossible without the use of mobile devices.

\section{CONCLUSION}

As has been shown in the above examples and many more that are available in the literature, mobile devices really make a difference in education. Their possibilities allow defining new kinds of scenarios that improve motivation, increase retention, enhance creativity, facilitate more flexibility, etc. New architectures (like we have done in [12] some time ago) and new settings have to be defined to fully take advantage of these possibilities. We are convinced that we will see many more applications in the years to come where mobile devices will make education a completely new experience.

\section{ACKNOWLEDGMENT}

The eMadrid Excellence Network is being funded by the Madrid Regional Government (Comunidad de Madrid) with grant No. S2009/TIC-1650. We wish to acknowledge stimulating discussions with our partners in the context of the network. Partial support has also been received from the Learn3 project (TIN2008-05163) and the EEE project (TIN2011-28308-C03-01). This is a summary paper that includes many developments carried out along many years and in many different settings. Many other people have contributed to the experiments carried out. In particular, apart from the authors of this paper, we would like to mention many interesting discussions within the Gradient research lab of the GAST research group at the Department of Telematics Engineering of UC3M and the participation of Julio Villena (UC3M) for the "creating a back-channel" experiment, Pedro MuñozMerino (UC3M) for the "sending learning pills" experiments, Mar Pérez-Sanagustín (then UPF, now UC3M), Davinia Hernández-Leo (UPF), Patricia Santos (UPF), Josep Blat (UPF), Luis de la Fuente (UC3M), and Gustavo Ramírez (then UC3M on leave from U. del Cauca, Colombia, now back there) for the "educational gymkhana" experiment, Álvaro Reina (then UC3M, now Galilea Soluciones) and Ángela di Serio (then UC3M on leave from U. Simón Bolívar, Venezuela, now back there) for the "augmented reality" experiments, and José Jesús García Rueda (UC3M), Derick Leony (UC3M), David Maroto (UC3M), Diego Morillo (UC3M), Selene Pinillos (then UC3M), Francisco Sánchez (UC3M), and the collaborators at Elecnor-Deimos, DNX, and UNED within the España Virtual project for the "cross reality" experiment, a project that was funded by the Ingenio 2010 programme, where we were subcontracted by Elecnor-Deimos.

\section{REFERENCES}

[1] N.A. Gromik: "Cell phone video recording feature as a language learning tool: A case study", Computers \& Education. 2012;58(1):223230. Available at: http://dx.doi.org/10.1016/j.compedu.2011.06.013 [Accessed 21 September 2011] 
[2] Israel Gutiérrez Rojas, Raquel M. Crespo García, Carlos Delgado Kloos: "Orchestration and feedback in lab sessions: improvements in quick feedback provision”, In: Towards Ubiquitous Learning. 6th European Conference of Technology Enhanced Learning, EC-TEL 2011 Palermo, Italy, September 20-23, 2011 Proceedings. LNCS. 2011; 6964

[3] Francis Heylighen, Clément Vidal: "Getting Things Done: The Science behind Stress Free Productivity”, Long Range Planning 41 (2008): 585605

[4] Immersive Education Initiative, http://mediagrid.org/ [Accessed November 2011]

[5] George A. Miller: "The magical number seven, plus or minus two". Psychological Review 63:2 (1956), pp 81-97

[6] M. G. Moore, G. Kearsley: "Distance education: a systems view", Belmont, CA. Wadsworth Publishing Co., 1996

[7] Mario Muñoz Organero, Pedro Muñoz Merino, Carlos Delgado Kloos: "Sending Learning Pills to Mobile Devices in Class to Enhance Student Performance and Motivation in Network Services Configuration Courses", IEEE Trans. Education, 2011, Vol. 99, pp. 1-5
[8] Mar Pérez-Sanagustín, Gustavo Ramírez-González, Davinia HernándezLeo, Mario Muñoz-Organero, Patricia Santos, Josep Blat, Carlos Delgado Kloos: "Discovering the campus together: A mobile and computer-based learning experience", Journal of Network and Computer Applications 35 (2012), pp. 176-188

[9] Álvaro Reina Nieves, Ángel Di Serio, Carlos Delgado Kloos: "Current and Prospective Role of Augmented Reality in Mobile Learning", X Jornadas de Ingeniería Telemática, JITEL 2011, Santander, 28-30 Sept. 2011

[10] Carol Rodgers: "Defining Reflection: Another look at John Dewey and Reflecting thinking". Teachers College Records, vol. 104, No. 4, pp 842-886

[11] G. Salomon, D. Perkings, T. Globerson: "Partners in Congnition: Extending Human Intelligence with Intelligent Technologies". Educational Researcher, 20(3), 2-9. Wittrock, MC (1974)

[12] Mario Muñoz Organero, Carlos Delgado Kloos: "Pervasive learning management system components and services for a pervasive m-learning architecture". International Journal of Mobile Learning and Organisation, 1(3), 275-287, 2007 